\title{
Problemáticas públicas como temáticas controversiales. Profesorado de historia y la enseñanza de la independencia nacional para la formación ciudadana en el Bicentenario de Chile
}

\author{
Public Issues as Controversial Issues. History and Education Teachers approaching National \\ Independence and Civic Education in the Chilean Bicentennial
}

\author{
Questões públicas como temas controversos. Professores de História e o ensino na \\ aborgadem da independência nacional e formação cidadã no Bicentenário do Chile
}

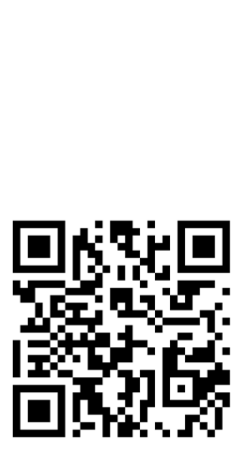

Eduardo Cavieres-Fernández

Universidad de Playa Ancha

Centro de Estudios Avanzados

Viña del Mar, Chile

ecavieres-cea@upla.cl

iD https://orcid.org/0000-0003-3199-5090

Ramón Alexander Uzcátegui-Pacheco

Universidad Andrés Bello

Viña del Mar, Chile

razktgui@gmail.com

https://orcid.org/0000-0002-5669-6663

Recibido • Received • Recebido: 14 / 10 / 2019

Corregido • Revised • Revisado: 11 / 12 / 2020

Aceptado • Accepted • Aprovado: 16 / 02 / 2021

Resumen:

Objetivo: El objetivo de este estudio es explorar las temáticas públicas que el profesorado chileno de historia, de enseñanza secundaria, estudiado en esta investigación, considera relevantes para ser discutidas durante la enseñanza de la independencia nacional (1810-1818) en vistas a la formación ciudadana de su estudiantado en el contexto del Bicentenario en Chile. Metodología: Para ello, se utilizan instrumentos cualitativos tales como la entrevista y observaciones de clase con participantes principales y entrevistas grupales con participantes secundarios provenientes de cuatro ciudades ubicadas a lo largo del país. Resultados: Este profesorado investigado coincide en señalar problemáticas que han supuesto relaciones de poder que afectan lo público y, ante las cuales, la enseñanza de la independencia nacional aporta elementos para reflexionar en profundidad sobre estos temas. Conclusiones: Si bien en las clases observadas al profesorado estudiado no hubo discusiones propiamente tales en torno a estas problemáticas, los intercambios iniciados por el personal docente sobre estas sí deben ser valorados, al menos, como un intento por hacerlas controversiales en contextos curriculares que no siempre permiten la discusión.

Palabras claves: Formación ciudadana; temas controversiales; profesorado; espacio público; independencia nacional. 
http://doi.org/10.15359/ree.25-2.3

http://www.una.ac.cr/educare

educare@una.ac.cr

\begin{abstract}
:
Objective: This study aims to explore the public issues that Chilean high-school history teachers consider relevant to be discussed during the teaching of the national independence (1810-1818) for their students' citizenship education in the context of the Chilean Bicentennial. Method: For this purpose, qualitative instruments, such as interviews and classroom observations, are conducted with main participants and group interviews with secondary participants from four cities throughout the country. Results: These teachers coincide in pointing out problems concerning power relations that affect the public sphere, before which the teaching of the national independence provides elements for in-depth reflections on these issues. Conclusions: Although there were no discussions around these problems in the classes observed with the teachers, the exchanges initiated by the teachers around these topics should be valued, at least, as an attempt to make them controversial in curricular contexts that do not always allow for discussion.
\end{abstract}

Keywords: Citizen education; controversial issues; teachers; public space; national independence.

\title{
Resumo:
}

Objetivo: O objetivo deste estudo é explorar as questões públicas que a história chilena e os professores do ensino secundário consideram relevantes para serem discutidas durante o ensino da independência nacional (1810-1818), tendo em vista a formação cidadã de estudantes no contexto do Bicentenário do Chile. Metodologia: Para isso, são utilizados instrumentos qualitativos, como entrevistas e observações de classe com participantes principais e entrevistas grupais com participantes secundários vindos de quatro cidades localizadas em vários lugares do país. Resultados: A equipe de professores coincide em apontar questões que envolveram relações de poder que afetam o público e, diante disso, o ensino da independência nacional fornece elementos para uma reflexão aprofundada sobre essas questões. Conclusão: Embora na observação das aulas com os professores não tenha havido discussões sobre estes problemas, as trocas iniciadas pelos professores sobre estes temas devem ser valorizadas, pelo menos como uma tentativa de provocar debate em contextos curriculares que nem sempre permitem a discussão.

Palavras-chave: Formação cidadã; questões controversas; professores; espaço público; independência nacional.

\section{Introducción}

En momentos en que diversos países en la región celebran su Bicentenario, diferentes cuestiones son materia de discusión entre la ciudadanía como la falta de participación política en la esfera pública. En Chile, y sin caer en reduccionismos, estos temas han permanecido desde el proceso mismo de su independencia (Pinto Vallejos y Valdivia Ortiz de Zárate, 2009). Por tanto, en el contexto de políticas educativas que buscan formar para la deliberación acerca de temas públicos, el Bicentenario ofrece un espacio para que el estudiantado discuta estas temáticas mientras estudia acerca del proceso de la independencia nacional (1810-1818). Esto coincide con lo prescrito en el currículo chileno que establece la enseñanza de la independencia nacional para octavo año básico, y en conexión con la promoción de valores y actitudes ciudadanas tales como el aprecio por la democracia, por una ciudadanía activa y participativa, la igualdad de derechos y el bien común (Ministerio de Educación de Chile, 2016).

2 
Lo anterior se vincula a los estudios sobre la discusión en el aula, en torno a temas controversiales, y su contribución a la formación ciudadana del estudiantado (Hess, 2009). Si bien estos estudios, incluyendo los de Chile, han señalado que el profesorado implementa con poca frecuencia discusiones sistemáticas en su enseñanza, también enfatizan la importancia que le dan a este tipo de pedagogía y a proponer asuntos a ser discutidos. En particular, este estudio busca explorar las temáticas de interés público que docentes de historia y ciencias sociales consideran relevantes para ser discutidas durante la enseñanza de la independencia nacional en vistas a la formación ciudadana del estudiantado en el contexto del Bicentenario. En el logro de este objetivo se atienden las siguientes preguntas de investigación: ¿Cuáles son las problemáticas públicas más relevantes para este profesorado? ¿Cómo contribuye el Bicentenario y la enseñanza sobre la independencia nacional a la discusión de estas problemáticas? Finalmente, ¿cuáles de estas problemáticas podrían constituir temas controversiales a ser discutidos durante la enseñanza de la independencia nacional?

Con el fin de desarrollar estas preguntas, la investigación utiliza instrumentos cualitativos para centrarse en las concepciones de cuatro profesores y profesoras de Chile pertenecientes a las ciudades de La Serena, Valparaíso, Concepción y Temuco, y se complementan sus contribuciones con observaciones en el aula y los aportes de otros profesores y profesoras. Para el análisis de estas concepciones, este trabajo se sustenta en un marco teórico conformado por estudios sobre las diversas temáticas que presentamos a continuación: la formación deliberativa del estudiantado y los temas controversiales, problemáticas de interés en el espacio público y su vinculación a la independencia nacional y la importancia del profesorado en la delimitación de temáticas de interés público a ser discutidas en el aula.

\section{Marco conceptual}

\section{Formación ciudadana, deliberación y temas controversiales}

Durante las últimas décadas, a nivel mundial y latinoamericano, las políticas educativas del sistema escolar han promovido un renovado énfasis acerca de la importancia de preparar al estudiantado para el ejercicio de la ciudadanía (Levinson y Berumen, 2007). Así, en Chile, las directrices curriculares han reforzado la implementación de esta área de aprendizaje con nociones de ciudadanía activa que comprometen a la ciudadanía en la defensa del sistema democrático y la institucionalidad política, y en su permanente perfeccionamiento ante los múltiples factores nacionales e internacionales que la ponen en riesgo (Ministerio de Educación de Chile, 2016). Aquello supone estrategias de enseñanza para que el estudiantado adquiriera información y plantee sus opiniones, de manera especial, en torno a temas controversiales o de interés público, atendiendo la diversidad e inclusión. 
http://doi.org/10.15359/ree.25-2.3

http://www.una.ac.cr/educare

educare@una.ac.cr

Los estudios sobre discusión en el aula coinciden en la importancia de enseñar al estudiantado a deliberar públicamente. Esta herramienta pedagógica permite al estudiantado conocer diversas perspectivas respecto a temáticas de carácter público y le ayuda a desarrollar el conocimiento, los valores, y las habilidades requeridas para una ciudadanía informada, deliberativa y participativa (Harrison, 2012). Por ello, esta discusión en el aula debe respetar los principios de la inclusividad y el respeto a las diferencias entre estudiantes, así como favorecer el libre intercambio de argumentos con una mayor comprensión de los temas discutidos y no la mera imposición de una perspectiva sobre otra (Schuitema et al., 2018).

Por consiguiente, una temática controversial es un componente fundamental de la discusión en el aula, que involucra preguntas referidas al ámbito público y que están abiertas a una multiplicidad de respuestas contrapuestas que posibilitan la deliberación (Hess, 2009). También puede remitir a conflictos que dividen a la sociedad, los que, además, puede resultar de interés para el estudiantado, si le afecta directamente (López Facal y Santidrían Arias, 2011). De manera particular, el área curricular de la historia tiene una afinidad con estas temáticas, al centrarse en aquellos eventos significativos que moldean a las sociedades de acuerdo con los posicionamientos de poder que asumen los distintos grupos sociales y cuyas consecuencias continúan generando antagonismo en la sociedad (Barton y McCully, 2007). Por su parte, McAvoy y Hess (2013) se refieren a estas consecuencias como temas perennes que aún provocan polarización, y que involucran valores fundamentales para la sociedad como lo son la justicia, la distribución del poder o la igualdad.

Finalmente, la comprensión respecto de las temáticas controversiales asumida en este trabajo considera, además, el grado de controversialidad que pueda estar presente en el currículo mismo, en la medida que constituye una selección de contenidos que suele reforzar determinadas perspectivas por sobre otras (Apple, 2014). Así, por ejemplo, en Chile, las políticas sobre formación ciudadana que hemos reseñado más arriba, si bien favorecen una comprensión más activa de la ciudadanía, han sido cuestionadas por dar una mayor valorización a las instituciones políticas del Estado por sobre otros ámbitos de participación ciudadana (Cavieres-Fernández, 2017). Igualmente, Vallejos Silva (2016) plantea que, si bien los objetivos de formación ciudadana para el área de la historia, geografía y ciencias sociales del currículo chileno fomentan la vinculación con la comunidad y la sociedad civil, se acentúan más otras orientaciones también presentes que promueven la meritocracia individualista, en sintonía con las políticas económicas más amplias.

\section{Temas públicos e independencia nacional}

Los puntos anteriores se relacionan con las perspectivas teóricas centrales de este estudio que vinculan la participación ciudadana, específicamente la deliberación, con la sociedad civil y el espacio público, que es donde la ciudadanía delibera sobre sus asuntos comunes, en 
http://doi.org/10.15359/ree.25-2.3

autonomía del Estado (Edwards, 2004). Estas perspectivas también acentúan las dificultades que existen para el desarrollo de dicha participación, debido, precisamente, a las tensiones que se originan con el Estado (Cohen y Arato, 1992). De manera particular en Latinoamérica, la construcción del Estado favoreció la concentración de poder económico y político en grupos sociales de elite que se beneficiaban de las relaciones de dependencia que se establecían con Estados económicamente más robustos (Faletto, 2014). El resultado ha sido sociedades civiles relativamente débiles y disgregadas, vis a vis la conformación de Estados oligárquicos y autoritarios. No obstante, Dagnino (2011) advierte que, dado el surgimiento de una multiplicidad de movimientos sociales en la región, la sociedad civil no debiera autosegregarse más del Estado, sino que debiera procurar establecer nuevas formas para influir en el.

Históricamente, la sociedad chilena también se ha construido desde una matriz estatal que no ha permitido la conformación de una sociedad civil fuerte con la autonomía para articular sus demandas frente al Estado. En las últimas décadas, esta dinámica se ha reforzado por los gobiernos de turno que han favorecido políticas a favor de fortalecer el crecimiento económico del país. Estos proyectos han obstaculizado el espacio público en tres aspectos. En primer lugar, la inserción de la economía nacional en los mercados internacionales ha fortalecido a las elites y, con ello, su participación en el Estado, lo que se ha reforzado por el centralismo regional en el país (Medina Valverde y Guzmán, 2016; Oyarzún, 2013). Del mismo modo, se ha afectado la representatividad del sistema político haciéndole funcional a las demandas del mercado por sobre su deber de resguardar derechos fundamentales pertenecientes a la ciudadanía (Atria et al., 2013). Finalmente, a través de las políticas en curso, el Estado ha procurado establecer una relación colaborativa con la sociedad civil, pero más bien de carácter instrumental supeditada al logro de determinados índices económicos (Delamaza, 2009). No obstante, frente a ello, en los últimos años, diversos movimientos de protesta han emergido desde la sociedad civil para cuestionar el alcance de estas políticas (Rojas Hernández, 2012).

Del mismo modo, diversos estudios también han subrayado la similitud entre estos aspectos problemáticos de la sociedad chilena en el contexto de su Bicentenario y el proceso de la independencia nacional (1810-1818). Por ejemplo, mencionan su carácter elítico al haber sido liderada por un grupo criollo de la aristocracia local ligada a la actividad comercial y militar, lo que supuso la exclusión de variados grupos, como el bajo pueblo y los grupos indígenas, de las discusiones respecto de la construcción de la nueva nación (Pinto Vallejos y Valdivia Ortiz de Zárate, 2009). Por su parte, el bloque dirigente patriota no fue homogéneamente compacto, sino que estuvo entrecruzado por disputas internas relativas al modo de gobierno que debía adoptarse y a temas de representatividad (Cavieres Figueroa, 2012). De la misma forma, los grupos patriotas de provincia discreparon del mayor protagonismo ostentado por los patriotas de Santiago y, en general, a través de sus cabildos, posibilitaron localmente una mayor participación entre su ciudadanía en comparación al proceder autoritario de la dirigencia 
http://doi.org/10.15359/ree.25-2.3

http://www.una.ac.cr/educare

educare@una.ac.cr

santiaguina (Salazar, 2011). Fue igualmente controversial la presencia del general argentino José de San Martin, considerado por algunos como un elemento foráneo (Lynch, 2009). En definitiva, los actuales procesos políticos poseen elementos de continuidad con la independencia nacional, en cuyo centro se ubican, de manera perenne, los aspectos problemáticos que subsisten en los modos cómo se participa públicamente en el país. Este asunto tiene profundas implicancias pedagógicas para el profesorado, las que se analizan a continuación.

\section{La contribución del profesorado a los temas controversiales en el aula}

Los estudios en el área subrayan la importancia del profesorado en la inclusión de temas controversiales en el aula. Específicamente, describen su rol en la implementación de discusiones en el aula, lo que implica crear un ambiente para que el estudiantado exprese sus diferentes perspectivas y haga conexiones con los contenidos disciplinares del currículo (Hess, 2011). Al respecto, el profesorado debe enfrentar una serie de dificultades y desafíos que incluyen la falta de tiempo con la que disponen para este tipo de actividades, debido a la presencia de prescripciones curriculares que acentúan otro tipo de aprendizajes; y la diversidad de intereses y niveles de participación que existe entre el estudiantado (Avery et al., 2013).

Dentro de los mayores desafíos también está la selección de un tema controversial para ser discutido, aspecto particularmente importante en este trabajo. En esta elección incide el compromiso del profesorado hacia la enseñanza de habilidades ciudadanas, y en el contexto de la enseñanza de la historia, hacia la realidad sociocultural del estudiantado, lo que permite moldear discusiones en torno a hechos históricos que generan posturas contrarias en el aula (Kello, 2016). Particularmente desafiante para la comunidad docente son aquellos temas que tienen implicancias locales o personales y en los cuales se tienen posturas propias definidas, pero que, dada su relevancia pública, deben ser 'abiertas' para que puedan ser discutidas desde una diversidad de perspectivas (Swalwell y Schweber, 2016). Además, el profesorado debe sortear aquellos planteamientos curriculares del Estado que predefinen posiciones frente a estos temas o excluyen aquellas temáticas que producen criticas significativas a la institucionalidad política (Niens et al., 2013).

En Chile, las investigaciones también subrayan la importancia de las concepciones del profesorado de historia y ciencias sociales en la formación ciudadana escolar, las que transitan por comprensiones que integran el componente moral, la participación o el sentido crítico (Martínez-Rodríguez et al., 2019). Por contrapartida, los estudios señalan que la comunidad docente tiende al uso de metodologías más bien expositivas por sobre estrategias interactivas que favorezcan la participación del estudiantado (Bonhomme et al., 2015). Investigaciones específicamente referidas a la discusión sobre temas controversiales en el aula confirman esta percepción, al señalar que el profesorado no promueve el debate con frecuencia en el aula 
(Toledo Jofré, 2015). No obstante, el estudio de Cavieres Fernández (2015) menciona algunas dificultades curriculares e institucionales que el profesorado tiene para implementar este tipo de discusiones en torno a temáticas controversiales actuales de interés público y referidas a las políticas públicas implementadas en las últimas décadas en el país. Es precisamente el interés por conocer, en mayor profundidad, los contenidos de estas temáticas y su vinculación con la enseñanza de la independencia nacional en el contexto del Bicentenario lo que constituye el objetivo de este estudio.

\section{Metodología}

La investigación tiene como objetivo explorar, con instrumentos cualitativos, aquellas temáticas de interés público que docentes de historia consideran relevantes para ser discutidas durante la enseñanza de la independencia nacional en vistas a la formación ciudadana del estudiantado en el contexto del Bicentenario. De manera particular, el estudio se enfoca en las concepciones de cuatro docentes de historia de enseñanza media, complementadas con observaciones en el aula y los aportes de otros profesores y profesoras.

\section{Participantes}

Para la selección de participantes, se invitó a profesores y profesoras con más de 5 años de experiencia y pertenecientes a establecimientos escolares públicos o subvencionados (privados, pero financiados por el Estado) con el supuesto de que, por un lado, el estudiantado de estos establecimientos son los más afectados por problemáticas públicas (i.e. segregación, desigualdad, etc.) y, por tanto, habría más discusión sobre estas temáticas en sus aulas. Por otro lado, dado lo complejo de implementar discusiones en el aula, el profesorado que hubiese superado una etapa inicial de inducción tendría más éxito implementando esta metodología. Los datos de contacto de este profesorado participante se obtuvieron fundamentalmente a través de consultas con personal académico de programas de formación docente, y, en promedio, se enviaron 20 invitaciones por cada ciudad involucrada en esta investigación. Las personas participantes son exclusivamente aquellas que respondieron positivamente a la invitación.

En esta investigación, se incluyen participantes principales, que constituyen el foco central del estudio, y participantes secundarios, que complementan los aportes entregados por el grupo principal. En términos geográficos, las profesoras y los profesores participantes provienen de las siguientes ciudades: Valparaíso (por representar el centro de Chile en donde se decidió la independencia del país), La Serena y Concepción (dado su protagonismo como provincias durante el proceso independentista); y Temuco (por representar a la población indígena existente durante dicho periodo). De este modo, la investigación contó con un 
http://doi.org/10.15359/ree.25-2.3

http://www.una.ac.cr/educare

educare@una.ac.cr

participante principal hombre y tres secundarios (dos mujeres y un hombre) de La Serena; con uno principal hombre y dos secundarios (un hombre y una mujer) de Concepción; con un participante principal mujer y tres secundarios (dos hombres y una mujer) de Valparaíso, y con un participante principal mujer y dos secundarios (un hombre y una mujer) de Temuco.

Entre agosto del 2018 y marzo del 2019, se combinaron secuencialmente técnicas cualitativas de recolección de datos. Así, en cada ciudad, se partió observando al sujeto participante principal durante dos clases en las cuales enseñaba la unidad sobre la independencia de Chile que el currículo nacional prescribe para el octavo año básico. Estas observaciones duraron una hora y media cada una y fueron registradas con grabadora de audio y notas de campo. A continuación, se entrevistó a cada participante principal para consultarle sobre aspectos contextuales como su establecimiento educativo, sus estudiantes, el programa que debe implementar, y metodologías de enseñanza, entre otros. Luego, en cada ciudad se realizó una entrevista grupal con el sujeto participante principal y los secundarios para abordar temas tales como las problemáticas y los espacios de deliberación en sus ciudades, la relevancia del Bicentenario ante estas problemáticas, la relación entre la enseñanza de la independencia nacional y la formación para la deliberación, y las temáticas controversiales referidas a la independencia a discutir en el aula. Finalmente, se realizó una entrevista con cada participante principal para ahondar las perspectivas abordadas en la entrevista grupal. Todas las entrevistas fueran grabadas con registro de audio y transcritas íntegramente.

\section{Análisis de datos}

Para el análisis de los datos se utilizaron técnicas cualitativas tradicionales. Las entrevistas individuales y grupales, y las observaciones de clases fueron codificadas por separado y por ciudad. De este modo, para cada ciudad, las entrevistas individuales se constituyeron en fuentes principales, mientras que las observaciones en el aula y las entrevistas grupales en fuentes complementarias. En primer lugar, se seleccionaron de las observaciones exclusivamente aquellos segmentos que contuvieran una interacción entre el profesorado y sus estudiantes en torno a un tema que vinculara temáticas actuales de interés público e independencia nacional. Luego, estos segmentos fueron codificados de acuerdo con dichos temas. En segundo lugar, se codificaron las entrevistas individuales haciendo uso de los códigos que emergieron del análisis de las observaciones de clases y otros derivados del marco conceptual de este trabajo. Así, algunos de los códigos fueron: enseñanza de la independencia nacional, temas públicos, espacio público, problemáticas públicas, discusión en el aula, estudiantado, interacción en el aula, etc. En tercer lugar, a través de nuevos ciclos de codificación, los códigos que se relacionaban entre sí fueron subsumidos en categorías temáticas englobantes que permiten dar respuestas a las preguntas de investigación. Por último, las entrevistas grupales se codificaron utilizando los mismos códigos de las entrevistas individuales. 
Finalmente, para reportar los hallazgos, para cada ciudad, se elabora un relato con un foco particular puesto en la contribución del respectivo participante principal. Esta contribución es complementada con aportes relevantes de los correspondientes sujetos participantes secundarios, y con datos pertinentes analizados a partir de las observaciones de clases. Para mantener el anonimato del grupo de participantes principales, se utilizan seudónimos. Para el caso del grupo secundario de participantes se utiliza una nomenclatura conformada por la inicial de la ciudad a la que pertenecen ( $, V, C$ o T); la inicial de su sexo $(\mathrm{H}$ o $\mathrm{M})$ y, en caso de que haya dos participantes del mismo sexo, un número (1 o 2).

\section{Resultados}

\section{El profesor de La Serena y el elitismo}

Para el profesor José, la problemática pública que más le inquietaba era el elitismo en el sistema político. Este aspecto se apreciaba en los proyectos de modernización que el Estado había realizado en La Serena, pero sin generar vínculos con la ciudadanía, tal como lo describía otro profesor $(\mathrm{SH})$ ante el problema de urbanización que afectaba a la ciudad: La autoridad no quiso prever este crecimiento y no fue dando soluciones, o dio soluciones parche. Entonces la gente ¿Dónde va? ¿A quién reclama? Si bien una profesora (SM1) señalaba que en la ciudad hay manifestaciones por diversos temas, (y) una conciencia de que yo puedo reclamar, yo tengo derechos, yo puedo expresarme; en el sentir de José, los ciudadanos no generan una ruptura con el Estado, es poca la crítica; en grupo si, por ejemplo, nosotros los profesores, pero la identidad serenense es muy ligada al Estado. José también veía reflejada esta falta de sentido crítico en sus estudiantes: están muy alejados de la realidad país, dado que no hay mucho debate o reflexión en torno a lo que sucede en la comuna, en la región, en el país, etc. Son bastante alejados en ese sentido.

De igual modo, la celebración del Bicentenario en La Serena también fue elitista. Como lo señalaba un profesor (SH1): Es un tema tan de elite ¿En que se basó el bicentenario en nuestro país? en obras, construcciones, inauguraciones, pero (no en) algo mas allá en que la gente se identificara como país. Para José, este elitismo se remontaba a la misma independencia: Si hubiese sido en el siglo 20, hubiese sido mucho más consciente. Tocó una independencia del siglo 19 y fue de la elite. Las elites son las que piensan, pero el bajo pueblo no tiene incidencia en ella. En ese contexto, los profesores y profesoras participantes consideraban importante discutir acerca del significado que tenía la independencia, tal como lo sugirió una profesora (SM2): ¿Qué es independencia? ¿Qué significó en el momento? ¿Qué significa hoy para nuestro país? Estas preguntas, José las vinculó a otras de mayor actualidad: Como país, ¿qué estamos celebrando? Algunos dijeron 'la libertad', pero ¿qué libertad? O 'estamos celebrando 200 años de desarrollo como país' ¿y donde se observa ese crecimiento? Consecuente con todo ello, José relativizaba igualmente el valor que tiene la independencia nacional para discutir temas de actualidad en el aula: los estudiantes enganchan harto con el siglo 20, en adelante, por ejemplo, las grandes huelgas obreras. Ahí hay más 
http://doi.org/10.15359/ree.25-2.3

http://www.una.ac.cr/educare

educare@una.ac.cr

pertinencia, ... el hecho de enseñar patria vieja, nueva, reconquista (las etapas de la independencia) no tiene mucha importancia para la comprensión de los sucesos actuales.

Respecto de sus clases, al profesor José le gustaba integrar a sus estudiantes: cuando los estudiantes participan es cuando pongo ejemplos cotidianos de lo que está pasando en el país. Si bien están como bien alejados de eso, siempre tienen algo que decir. En particular, durante la enseñanza de la independencia: en todas las temáticas y discusiones aparecía la elite y, por eso, me interesa que los chiquillos lo entiendan. Así, en las clases observadas, al ver las causas de la independencia, en varias ocasiones pidió al estudiantado que expresaran su preferencia entre un sistema político liderado por un rey y otro por un presidente, a modo de subrayar los peligros de que solo una persona decidiera por los intereses de todas las demás. Con ello, comprobó que los estudiantes y las estudiantes valoran el sentido de la libertad. Recuerdo harta discusión con la monarquía. Yo les dije, ¿a ustedes les gustaría volver a ser una monarquía? No, dicen ellos, porque no va en la genética de los latinoamericanos. No obstante, no hubo una discusión propiamente tal con una variedad de perspectivas. Si bien José reconoció que el texto escolar es bastante complementario, tiene varias visiones; primó su afán de que el estudiantado aprendiera lo que deseaba enseñarles respecto del tema. Como lo confesó después: Es tanto mi ímpetu ... que no le doy interés (a lo que los estudiantes) quieren decir y termino con lo que yo digo. A pesar de ello, también influyó la presión por pasar la unidad dentro del tiempo estipulado por el programa escolar, lo que le llevó a focalizarse en aspectos fundamentalmente conceptuales.

\section{El profesor de Concepción y la dependencia}

Desde la perspectiva del profesor Joel, el tema de la dependencia de Concepción con el centro del país, en donde está la capital Santiago, era una problemática pública importante, tal como lo explicaba otro profesor (CH): apoyamos nuestra independencia de Santiago, que somos como separatistas de Santiago, que odiamos a Santiago, pero en el fondo dependemos mucho de Santiago. Como agregó Joel, todo era para Santiago, los conciertos, las mejores bibliotecas están en Santiago, los mejores colegios están en Santiago. Ello influía en los problemas de viviendas y de medio ambiente que afectaban a la ciudad y que no habían sido resueltos por los gobiernos de turno más preocupados por los problemas en el centro del país. Frente a ello, de acuerdo con el mismo profesor anterior $(\mathrm{CH})$, se habían mantenido en el tiempo ciertas tradiciones de organización social en la ciudad y en las cuales hay un acto de ciudadanía de participar y tomarse los espacios. No obstante, de acuerdo con Joel, también habían aparecido signos de pasividad en la ciudadanía al sentir que no puede influir en el Estado.

Respecto de la celebración del Bicentenario en Concepción, esta se redujo a un evento sin mayor impacto, tal como lo describe una profesora (CM): la gente iba porque había un show, había artistas, porque iban a tirar fuegos artificiales en el río. En el caso de Joel, señaló que lo recordé en la sala, que habíamos cumplido los 200 años, pero aparte de eso ni un acto. Los colegios 
además funcionan por orden ministerial, entonces no mandaron nada del Ministerio (ubicado en Santiago). A ello, la profesora (CM) agregó que, aunque haya un currículo escolar para el país, para Concepción, como ciudad que tiene una importancia relevante para la independencia, se debiese tomar otros contenidos, tener otros ejes temáticos que sean relevantes para la ciudad. Ello permitiría ofrecer perspectivas importantes para la formación ciudadana del estudiantado considerando que varios próceres que provinieron de Concepción querían cambiar una política que ya estaba colapsada y que como ciudadanos se sintieron con el derecho de decir 'no más'; cosas que nosotros hemos como perdido. Según el otro profesor $(\mathrm{CH})$, todo ello ayuda a discutir, si la independencia de Chile contribuyó de verdad a la participación ciudadana:

algunos dicen que nunca logró ser independiente, que seguía respondiendo a los intereses económicos extranjeros, que la elite siempre llevo adelante todas las decisiones políticas. Otros dicen que no, que si había un cabildo, que se podía opinar y decidir.

Por su parte, el profesor Joel señalaba que, en sus clases, un tema de historia que estemos pasando, trato de llevarlo a la actualidad porque ahí los chicos lo entienden y empiezan a opinar. No obstante, advertía sobre las dificultades que tenía para implementar discusiones en el aula: Para eso hay que preparar al chico, tiene que ser consciente de que tiene que tener información. Entonces es muy complicado con mis alumnos, porque a muchos no les gusta la historia. Consecuentemente, en las clases observadas, hubo poca interacción entre él y el estudiantado, con un foco más bien centrado en los contenidos, tal como eran presentados en el texto escolar, y exigidos por su colegio. No obstante, Joel intercaló comentarios referidos a la problemática de la dependencia, como el siguiente:

A pesar de ser un país democrático con un libre comercio ... dependemos de otros países; y agregó: Nuestro gran ejemplo es Norteamérica; si algo es boom o moda en Norteamérica nosotros lo copiamos. Los bailes que ustedes hacen ¿de dónde vienen?

Si bien preguntas como estas no llevaron a una discusión más amplia, Joel consideraba esta temática fundamental: el tema es si realmente pasamos o no a ser un territorio independiente. Sin ningún atajo o ningún control externo. Y si no es así, ¿qué hacer como ciudadanos para poder hacerlo? Con este tipo de temas, Joel buscaba que sus estudiantes pudieran argumentar, tuvieran la posibilidad de criticar. Porque veo a algunos de ellos que están como demasiado dormidos y siento que la sociedad necesita nuevas ideas. Ante ello enfatizó que: por eso siempre trato de sacarles una opinión o algo así.

\section{La profesora de Valparaíso y la falta de participación directa}

Desde la perspectiva de la profesora María, la falta de participación directa era un problema público significativo que afectaba a Valparaíso. Según su descripción, en la ciudad no existía la 
http://doi.org/10.15359/ree.25-2.3

http://www.una.ac.cr/educare

educare@una.ac.cr

influencia directa si una inmobiliaria quiere instalar un edificio tapando a toda la población; o si quieren generar un parque deportivo, la población todavía no logra decidir eso que va a influir de forma directa en sus vidas. De acuerdo con otra profesora (VM), esto también se aplicaba a una mayoría de sus estudiantes:

No sienten que sean participantes de los cambios ... se identifican mucho con Valparaíso, pero no se hacen parte de cambiar la ciudad. La crítica siempre está, pero ser parte de la construcción de su barrio y de su población no.

No obstante, en Valparaíso pervivían muchos espacios de participación de donde habían surgido muchos movimientos políticos y sociales. Como lo subrayaba uno de los profesores (VH1): el ámbito artístico y cultural es sumamente potente. De hecho, en la opinión de María la gente está nuevamente haciéndose presente por participar en sus derechos, por exigirlos.

La celebración del Bicentenario tampoco tuvo mayor impacto tal como lo recuerda uno de los profesores (VH2), hace poco fue la conmemoración de los 200 años de zarpe de la flota libertadora (que partió de Valparaíso para liberar al Perú). Fue bonito, pero el trasfondo histórico pasó sin pena ni gloria. Tal como lo argumentó el otro profesor ( $\mathrm{VH} 1)$, aquello reflejaba la experiencia de Valparaíso, puerto desde donde se expandió la revolución independentista hacia el resto de Latinoamérica, pero cuya participación posterior quedo suprimida: Entonces hablar de esta tradición de revolución y contrarrevolución, está muy latente aquí en Valparaíso ... porque, ¿qué pasó con Valparaíso a posteriori? Llegaron estas oligarquías que hacen y deshacen en Chile. De ahí que, para este profesor ( $\mathrm{VH} 1$ ), la independencia nacional contenía un elemento muy cuestionable: íbamos para allá y mira donde llegamos y adónde vamos. Este aspecto le resultaba particularmente difícil a María al enseñar la independencia nacional:

Nosotros trabajamos con (estudiantes) de una clase trabajadora, ¿Cómo les mostramos que este proceso emancipador, si bien parte desde la cúpula, ellos también pueden ser participe? ... Que ese proceso no es muy alejado de la realidad de ellos.

De ahí que para la otra profesora (VM) fuese tan importante establecer vinculaciones entre la independencia y temáticas ciudadanas de Valparaíso, ya que permitía al estudiantado visualizar que esos conceptos, que son de mucho tiempo, muy antiguos, están presente todavía y son parte de una realidad cotidiana. No los vean tan ajeno.

Consecuentemente, para la profesora María era importante que sus estudiantes lograran trasmitir, expresar o crear cosas nuevas a partir del proceso de independencia. Que pudieran proyectar a la realidad de hoy ciertos procesos que se dieron en la época. No obstante, el enfoque del texto escolar no siempre era de ayuda: 
Se basa mucho en la Revolución Francesa, la independencia de las Colonias, más que en las características de la Independencia de Chile ... ¿En qué momento (los estudiantes) van a decir que son parte del proceso?

Sin embargo, en las clases observadas, hubo contenidos que permitieron intercambios breves como el referido a la participación de los sectores populares, y durante el cual, María intentó que sus estudiantes hicieran vinculaciones con su propia participación: ¿Les importó a los sectores populares el proceso de independencia? A ustedes hoy día, ¿realmente les interesa cuál presidente sale elegido en Chile? Las respuestas pudieron haber derivado en discusiones con posturas antagónicas en torno a planteamientos que incidían en la participación directa de las estudiantes y los estudiantes, como la falta de interés político en parte de la sociedad chilena o la limitada injerencia de los sectores populares actuales, restringida al sufragio. María lamentó no haber podido profundizar más estos temas, pero como lo describió, hubo un pequeño grupo y ahí generaron discusión, y siguieron la discusión, pero el resto, en su mayoría, (permaneció) en la indiferencia.

\section{La profesora de Temuco y el conflicto social}

Para la profesora Ana, la problemática pública de mayor relevancia en Temuco se refería al conflicto social. Si bien este conflicto estaba altamente influido por la presencia del pueblo mapuche en la zona, de acuerdo con Ana el conflicto viene desde hace muchos años, también hay otras demandas que el Estado no ha sabido dar suficientes respuestas. Como lo sintetizaba la otra profesora (TM): acá hay mucho abuso, entonces se genera una desconfianza. Problemas de pobreza extrema, drogas y un crecimiento urbano sin planificación ha llevado al deterioro de las condiciones de vida de la ciudad. Frente a ello, la misma profesora (TM) explicaba: estamos al margen, como que uno ve cómo pasan las cosas, pero nadie discute. Al respecto, otro profesor (TH) señaló que las autoridades no promovían la participación ciudadana:

hoy día se incorpora ... la participación ciudadana disfrazada, porque se da la firma y se aprueba y echamos a andar. Es más una 'avallasadora' de políticas que nosotros tomando una decisión de cómo queremos construir nuestro territorio.

Desde la perspectiva de Ana, la celebración del Bicentenario fue un evento de la clase política que simplemente reflejó lo que fue el proceso de la independencia:

Las personas, en general, no participaron; al pueblo mapuche se le utilizó para pelear con los españoles, pero después va quedando como renegado. Porque la clase política se olvida de esa libertad, de esa igualdad, que intentaron inculcar. 
http://doi.org/10.15359/ree.25-2.3

http://www.una.ac.cr/educare

educare@una.ac.cr

Como complementó el profesor (TH):

Si se piensa en el bullicio del cabildo sabiendo que eran un grupo chiquitito y que fueron diseñando lo que nosotros vivimos como país..., entonces ese momento sirve para colocar el tema en la mesa, que los ciudadanos siempre hemos estado afuera.

En ese sentido, las dos profesoras mujeres participantes reconocían la importancia de enseñar la independencia nacional, porque permitía reflexionar sobre la importancia de que en Chile se haya dado un Estado para proteger los derechos y satisfacer necesidades básicas como la educación o salud. También percibían el contraste entre ese ideal y la realidad, el que era apuntado por el mismo estudiantado. Como lo explicó Ana:

Es difícil porque uno habla de estos conceptos y los chicos son muy escépticos. Ellos en seguida lo relacionan con políticos y corrupción y uno les dice que no debería ser así, pero ellos no lo creen, no les interesa o lo ven con distancia.

Consiguientemente, la profesora Ana consideraba importante discutir con sus estudiantes si se había alcanzado el ideal de la elite de crear un orden estable o si en realidad todavía tenemos conflictos dentro del país. Respecto de las clases observadas a la profesora Ana, estas eran interactivas, pero más bien centradas en los contenidos conceptuales referidos a la independencia nacional -dada la presión sobre ella para que preparara al estudiantado para las pruebas estandarizadas-. El interés de Ana también estuvo puesto en que su estudiantado comprendiera el evento propiamente tal de la independencia, más que en hacer vinculaciones con problemáticas públicas actuales: el estudiante tiene que tener esa capacidad de comprender qué sucedía en esa época y cuáles fueron las repercusiones que tuvo en su momento y que las traen hasta el día de hoy. Por ello, una actividad de interés que solicitó a las estudiantes fue que se identificaran con alguna criolla de la época, pensaran en su participación durante el periodo de independencia y si aquella sería moderada o exaltada como se distinguió entre los criollos. Para Ana, la importancia de la actividad radicaba en que les cuesta a las estudiantes ver el pasado con los ojos del pasado, porque a veces uno ve el pasado a la luz del presente, y (la actividad) generó discusión. A la siguiente clase, el estudiantado analizó grupalmente la tarea, y concluyeron que, si hubieran sido una mujer de la época, hubieran sido tal vez unas criollas exaltadas. Según Ana, lo discutido coincidió con discusiones que se daban en el espacio público chileno. Por ello, ella pensaba que era muy probable que en el futuro promoviera discusiones en el aula con vinculaciones más explicitas entre la independencia nacional y conflictos del presente, porque aún tenemos algunas formas de opresión que están como bien solapadas. 


\section{Discusión}

Como ha sido señalado anteriormente, el presente estudio tenía como objetivo explorar las temáticas de interés público que docentes de las áreas de historia, geografía y ciencias sociales consideran relevantes para ser discutidas durante la enseñanza de la independencia nacional, en vistas a la formación ciudadana del estudiantado en el contexto del Bicentenario. A continuación, se discuten sus resultados a la luz de las preguntas que derivan de su objetivo principal.

\section{Problemáticas públicas relevantes para el profesorado}

Entre lo compartido por los profesores y las profesoras participantes de este estudio, se aprecian coincidencias en las temáticas públicas que consideran relevantes y que están situadas en un mismo horizonte dentro del país, en el cual se han desarrollado relaciones de poder entre el Estado y la ciudadanía que han afectado, decisivamente, la conformación de lo público, tal como se adelantó en el marco conceptual. Los problemas que señala este grupo participante describen fenómenos similares que se han desarrollado en las respectivas ciudades a las cuales pertenecen y que tienen incidencia en el modo de participar de la ciudadanía. En términos generales, estas problemáticas no hacen alusión a hechos específicos o contingentes, sino que aluden a formas estables presentes en la sociedad chilena y que remiten a los temas que en el marco conceptual son denominados como perennes (McAvoy y Hess, 2013), y que están vinculados a valores públicos como la igualdad, la democracia y la justicia. En el caso de este grupo docente, estos temas se vinculan a la ausencia de dichos valores: elitismo, dependencia, falta de participación directa y conflicto social.

No obstante, estas temáticas contienen un elemento conflictivo que supone una complejidad. Los profesores y las profesoras participantes describen un escenario en donde, por un lado, está la ciudadanía -entre los que se ubican ellos y ellas- y, por otro, el Estado o las elites. Dicha descripción pareciera responsabilizar fundamentalmente a una sola parte del conflicto: a quienes ostentan el poder. La complejidad de aquello no radica necesariamente en su validez que en lo sustancial es compartido por el marco conceptual de este trabajo-, sino en la dificultad que se pudiese generar para que esta perspectiva sea'abierta' a diferentes valoraciones (Swawell y Schweber, 2016) respecto a las causas o las responsabilidades que sustentan las problemáticas sociales (por ejemplo, que la ausencia de participación no solo se deba al autoritarismo de las elites, sino también a la pasividad ciudadana). Desde esta perspectiva, la formulación misma de estas temáticas por parte de los profesores y las profesoras participantes es problemática, en la medida en que se comprendan como versiones únicas de la realidad, sin permitir que algunos de sus aspectos sean considerados controversiales y posibiliten la discusión propiamente tal en el aula. Esta complejidad sería mayor, si estuviese reflejando una polarización presente en el contexto nacional más amplio, en donde los grupos sociales suelen promover relatos unilaterales que llevan a la división y no la deliberación. 
http://doi.org/10.15359/ree.25-2.3

http://www.una.ac.cr/educare

educare@una.ac.cr

\section{Discusión de problemáticas públicas e independencia nacional}

El profesorado investigado en este estudio sitúa el carácter perenne de las problemáticas sociales actuales en conexión con la independencia nacional. Tal evento es significado como un punto de origen para entender y discutir algunos de los conflictos que habiéndose hecho presentes en ese momento, aún persisten en la sociedad chilena. Desde esa perspectiva, no es de menor importancia que la celebración misma del Bicentenario, tal como fue experimentada por el grupo de participantes, refleje la distancia que existe entre el Estado chileno y la ciudadanía. Conforme a esta idea, si bien, en cuanto hecho histórico, se podría argumentar su distancia subjetiva respecto de los intereses del estudiantado -tal como lo manifestó el profesor José de La Serena- dicha distancia queda mediada precisamente por la vinculación con problemáticas actuales. De este modo, si bien la independencia nacional, con los acontecimientos que transcurrieron durante ella, pueda ser cuestionada como acontecimiento histórico o el modo cómo se enseña en el currículo, es más bien su vinculación con temas actuales lo que, finalmente, le da relevancia para la formación ciudadana del estudiantado, tal como lo planteó una profesora de Concepción. Desde esa perspectiva, en el parecer del profesorado que participó en este estudio, el componente ciudadano está presente al enseñar la independencia nacional.

Consecuentemente, el profesorado participante en esta investigación concuerda en la importancia de explorar preguntas con sus estudiantes respecto al proceso independista, las cuales son relevantes para temáticas que inciden en la formación ciudadana hoy, como lo sería, si la independencia alcanzada realmente fue tal, o, si fue realmente participativa. En principio, estas preguntas posibilitan posturas contrarias a favor y en contra que contribuyen con análisis más complejos para que el estudiantado conozca, en profundidad, el proceso independista, sus contribuciones y falencias, y deduzca, a su vez, elementos teóricos para una mejor comprensión de los problemas actuales. En la medida en que esta deliberación remite a un evento histórico distante subjetivamente del estudiantado, podría ayudar a establecer matizaciones en aquellas problemáticas actuales cuya proximidad histórica, al ser estudiadas directamente, producen polarización y no deliberación. Sin embargo, una vez más, aquello dependerá de si el profesorado presenta estos acontecimientos históricos como controversiales, y no solo reforzando una valoración a priori.

\section{Independencia y controversialidad en el aula}

Finalmente, para analizar la relación entre temáticas públicas, independencia nacional y temas controversiales a ser discutidos en el aula, es necesario reiterar que en las clases observadas a los profesores y las profesoras de La Serena, Valparaíso, Concepción y Temuco no se presenciaron discusiones, ya sea planificadas o surgidas en el momento, en torno a un tema controversial con posturas opuestas claramente definidas. Al igual como se planteó en el 
marco conceptual, ello se debió, en sus propias palabras, a la falta de interés del estudiantado por este tipo de actividad; o a la exigencia del currículo prescrito de pasar determinados contenidos conceptuales. En ese sentido, si bien estos profesores y profesoras formularon preguntas interactivas para que el estudiantado vinculara estos contenidos con temáticas de actualidad, el que no se haya hecho en profundidad a través de una discusión más sistemática podría haber representado la pérdida de una oportunidad (Journell, 2011), no solo para analizar detenidamente alguna temática, sino para promover una habilidad ciudadana, que pareciera no estar presente entre sus estudiantes y pareciera no estarlo tampoco en la sociedad chilena. No obstante, considerando el marco conceptual de este estudio, el desafío a futuro no pasaría solo por dar más cabida a estos intercambios, sino en elaborar más detenidamente las preguntas y comentarios referidos a estas temáticas públicas, para que, efectivamente, se propongan perspectivas diversas que puedan conducir a discusiones más extendidas en el aula.

Al respecto, y a la luz de los hallazgos, lo anterior implica, fundamentalmente, abrir las temáticas a una variedad de perspectivas más allá de las posiciones personales del profesorado. De esto no se concluye que el grupo de educadores estudiados deban limitarse a presentar neutralmente posturas antagónicas, sino que acentúen más el objetivo de que sus estudiantes cultiven miradas más matizadas y complejas respecto de problemáticas públicas de relevancia. Tampoco implica desconocer su esfuerzo por vincular los contenidos de la independencia con temáticas públicas, y no valorarlo como un punto de partida para generar en su estudiantado interés y reflexión, a través de preguntas que intentaron ofrecer perspectivas controversiales, al menos, entre lo que pensaban como profesores y profesoras y determinadas prácticas o discursos más dominantes presentes en la sociedad; más aún, considerando la presión sobre el personal docente para pasar determinados contenidos conceptuales que, al representar particulares comprensiones desde el Estado, pueden estar inhibiendo este tipo de aprendizaje ciudadano (Apple, 2014). Sin embargo, discutir críticamente en el aula acerca del elitismo, de la dependencia, la falta de participación directa y el conflicto social, a la luz de la independencia nacional, no solo requiere de preguntas que generen interacción. Junto a ello, se debe proponer al estudiantado un proceso de deliberación permanente y, en ello, podría ser fundamental cómo el profesorado formule estos temas para posibilitar una diversidad de perspectivas que superen la polarización que, precisamente, resulta de ellos.

\section{Conclusión}

El objetivo de este estudio era explorar las temáticas públicas que profesores y profesoras de historia consideran relevantes para ser discutidas durante la enseñanza de la independencia nacional en vistas a la formación ciudadana del estudiantado en el contexto del Bicentenario. Estos profesores y profesoras coinciden al señalar problemáticas perennes que han supuesto relaciones de poder que afectan a lo público; pero que, en la medida que en su formulación contienen 
http://doi.org/10.15359/ree.25-2.3

http://www.una.ac.cr/educare

educare@una.ac.cr

un elemento conflictivo, parecieran fomentar más la polarización que la deliberación. Ante ello, la enseñanza de la independencia nacional aporta elementos para reflexionar en profundidad sobre estos temas. No obstante, en las clases observadas a las profesoras y los profesores participantes, no hubo discusiones propiamente tales en torno a estas problemáticas, no solo dada ciertas situaciones contextuales, sino porque no fueron abiertas a posturas contrarias a ser discutidas. Sin embargo, los intercambios iniciados por este profesorado investigado en torno a estas temáticas sí deben ser valorados, al menos, como un intento por hacerlas controversiales en contextos curriculares que no siempre permiten la discusión.

Desde esa perspectiva, el estudio confirma la importancia del profesorado para promover la discusión en torno a contenidos curriculares que ayudan al estudiantado a comprender mejor la construcción del espacio público y preparase para participar en él. Específicamente, la enseñanza de la independencia nacional puede ayudar a analizar el elitismo, la dependencia, la falta de participación directa y el conflicto social que apuntan a una relación entre el Estado y la ciudadanía que tanto ha afectado al espacio público no tan solo en Chile, sino en los diversos países de Latinoamérica que también están celebrando sus Bicentenarios. No obstante, el cómo se formulen estas temáticas es de fundamental importancia, si con ellas se pretende generar espacios de discusión que ayuden a visualizar estos temas dentro de nuevos horizontes de compromiso y quehacer ciudadano. A su vez, aquello servirá para contrarrestar posturas polarizadas que no solo hacen más difícil la resolución de los conflictos, sino que no se condicen con los marcos democráticos y deliberativos que debiesen caracterizar la enseñanza de la independencia nacional para pensar en los nuevos espacios públicos que han de construirse en la región.

\section{Declaración de financiamiento}

Esta investigación ha sido realizada gracias al financiamiento de FONDECYT (Fondo Nacional de Desarrollo Científico y Tecnológico) proyecto N. ${ }^{\circ} 1180475$.

\section{Declaración de Material complementario}

Este artículo tiene disponible, como material complementario:

-La versión preprint del artículo en https://doi.org/10.5281/zenodo.4042044

\section{Referencias}

Apple, M. W. (2014). Official knowledge: Democratic education in a conservative age. Routledge.

Atria, F., Larraín, G., Benavente, J. M., Couso, J. y Joignant, A. (2013). El otro modelo. Del orden neoliberal al régimen de lo público. Debate. 
http://doi.org/10.15359/ree.25-2.3

Avery, P. G., Levy, S. A. y Simmons, A. M. M. (2013). Deliberating controversial public issues as part of civic education. The Social Studies 104(3), 105-114. https://doi.org/10.1080/003779 $\underline{96.2012 .691571}$

Barton, K. C. y McCully, A. W. (2007). Teaching controversial issues... where controversial issues really matter. Teaching History, 127(127), 13-19.

Bonhomme, M., Cox, C., Tham, M. y Lira, R. (2015) La educación ciudadana escolar de Chile 'en acto': Prácticas docentes y expectativas de participación política de estudiantes (pp. 373426). En C. Cox y J. C. Castillo (Eds.), Aprendizaje de la ciudadanía. Contextos, experiencias y resultados. Ediciones Universidad Católica de Chile.

Cavieres Fernández, E. (2015). Enseñando ciudadanía en medio del conflicto: Profesores de historia y movilizaciones estudiantiles en Chile. Revista Mexicana de Investigación Educativa, 20(67), 1311-1334. https://www.comie.org.mx/revista/v2018/rmie/index.php/ nrmie/article/view/168/168

Cavieres Fernández, E. (2017). Teacher counter stories to a citizenship education mega policy narrative. Preparing for citizenship in Chile. Journal of Curriculum Studies, 49(4), 414-436. http://dx.doi.org/10.1080/00220272.2016.1274783

Cavieres Figueroa, E. (2012). Sobre la independencia en Chile. El fin del antiguo régimen y los orígenes de la representación moderna. Ediciones Universitarias de Valparaíso.

Cohen, J. L. y Arato, A. (1992). Civil society and political theory. Massachusetts: The MIT Press.

Dagnino, E. (2011). Civil society in Latin America. En M. Edwards (Ed.), The Oxford handbook of civil society (122-132). Oxford University Press.

Delamaza, G. (2009). Tan lejos tan cerca. Políticas públicas y sociedad civil en Chile. LOM ediciones.

Edwards, M. (2004). Civil society. Polity.

Faletto, E. (2014). La especificidad del estado en América Latina. Estudios, 31, 205-236. https:// doi.org/10.31050/1852.1568.n31

Harrison, T. (2012). Assessing citizenship education: Challenges and opportunities. En J. Arthur y H. Cremin (Eds.), Debates in citizenship education (pp. 149-158). Routledge.

Hess, D. E. (2009). Controversy in the classroom. The democratic power of discussion. Routledge.

Hess, D. (2011). Discussions that drive democracy. Educational Leadership, 69(1), 69-73.

Journell, W. (2011). Teachers' controversial issue decisions related to race, gender, and religion during the 2008 presidential election. Theory and Research in Social Education, 39(3), 348392. https://doi.org/10.1080/00933104.2011.10473459 
http://doi.org/10.15359/ree.25-2.3

http://www.una.ac.cr/educare

educare@una.ac.cr

Kello, K. (2016). Sensitive and controversial issues in the classroom: Teaching history in a divided society. Teachers and Teaching: theory and practice, 22(1), 35-53. http://dx.doi.org/10.1080 /13540602.2015.1023027

Levinson, B. A. U. y Berumen, J. G. (2007). Educación para una ciudadanía democrática en los países de América Latina: Una mirada crítica. REICE. Revista Electrónica Iberoamericana sobre Calidad, Eficacia y Cambio en Educación, 5(4), 16-31. https://revistas.uam.es/reice/ issue/view/367

López Facal, R. y Santidrían Arias, V. M. (2011). Los "conflictos sociales candentes" en el aula. Íber: Didáctica de las Ciencias Sociales, Geografía e Historia, 69, 8-20. https://www.grao.com/es/ producto/revista-iber-067-enero-11-las-transiciones-dictadura-democracia

Lynch, J. (2009). San Martin. Argentine soldier, American heroe. Yale University Press.

Martínez-Rodríguez, R., Muñoz-Labraña, C., Sánchez-Agustí, M. (2019). What does it mean to be a citizen? A comparative study of teachers' conceptions in Spain and Chile. Education as Change, 23, 1-24. http://dx.doi.org/10.25159/1947-9417/4209

McAvoy, P. y Hess, D. (2013). Classroom deliberation in an era of political polarization. Curriculum Inquiry, 43(1), 14-47. https://doi.org/10.1111/curi.12000

Medina Valverde, C. y Guzmán, C. (2016). La invisibilidad del Biobío: Mirando el centro desde la periferia. Atenea, 514, 49-64. http://dx.doi.org/10.4067/S0718-04622016000200049

Ministerio de Educación de Chile (2016). Orientaciones curriculares para el desarrollo del plan de formación ciudadana. Autor. https://www.academia.edu/40628562/ORIENTACIONES CURRICULARES PARA EI DESARROLLO DEL PLAN DE FORMACI\%C $3 \% 93 \mathrm{~N}$ CIUDADANA

Niens, U., O'Connor, U y Smith, A. (2013). Citizenship education in divided societies: Teachers' perspectives in Northern Ireland. Citizenship Studies, 17(1), 128-141. https://doi.org/10.10 $\underline{80 / 13621025.2012 .716214}$

Oyarzún, L. (2013). When trade policy is not enough: Opportunities and challenges for Chile's international insertion. Journal of Iberian and Latin American Research, 19(2), 268-285. https://doi.org/10.1080/13260219.2013.853357

Pinto Vallejos, J. y Valdivia Ortiz de Zárate, V. (2009). ¿Chilenos todos? La construcción social de la nación (1810-1840). LOM ediciones.

Rojas Hernández, J. (2012). Sociedad bloqueada. Movimiento estudiantil, desigualdad y despertar de la sociedad chilena. Ril Editores. 
http://doi.org/10.15359/ree.25-2.3

http://www.una.ac.cr/educare educare@una.ac.cr

Salazar, G. (2011). Construcción de Estado en Chile (1800-1837). Democracia de los "pueblos". Militarismo ciudadano. Golpismo oligárquico. Editorial Sudamericana.

Schuitema, J., Radstake, H., van de Pol, J. y Veugelers, W. (2018). Guiding classroom discussions for democratic citizenship education. Educational Studies, 44(4), 377-407. https://doi.org/1 0.1080/03055698.2017.1373629

Swalwell, K. y Schweber, S. (2016). Teaching through turmoil: Social studies teachers and local controversial current events. Theory \& Research in Social Education, 44(3), 283-315. https:// doi.org/10.1080/00933104.2016.1201447

Toledo Jofré, M. I., Magendzo Kolstrein, A., Gutiérrez Gianella, V., e Iglesias Segura, R. (2015). Enseñanza de 'temas controversiales' en la asignatura de historia y ciencias sociales desde la perspectiva de los profesores. Estudios Pedagógicos, 41(1), 275-292. https://scielo. conicyt.cl/pdf/estped/v41n1/art16.pdf

Vallejos Silva, N. F. (2016). Supuestos y marcos conceptuales de formación ciudadana que subyacen en el currículum ministerial de historia, geografía y cs. sociales en la enseñanza básica chilena. Archivos Analíticos de Políticas Educativas, 24(45), 1-34. https://doi. org/10.14507/epaa.24.2235 